\title{
La dialisi extracorporea domiciliare: un'opzione terapeutica ancora auspicabile
}

\author{
Marco Gonella
}

\section{Divisione di Nefrologia e Dialisi, Ospedale Provinciale, Casale Monferrato (Alessandria)}

\section{$\mathbf{L}$}

a rivalutazione e il rilancio della dialisi extracorporea domiciliare (EDD) sono ampiamente condivisibili in termini di un ottimale rapporto costo/beneficio.

La EDD avrebbe dovuto avere, per una popolazione selezionata di pazienti, un'adeguata espansione in parallelo con altre strategie dialitiche quali la dialisi ad assistenza limitata e la dialisi ospedaliera serale o notturna (per i pazienti con attività lavorativa); nella realtà, queste ultime hanno quasi del tutto soppiantato la EDD, forse perché essa necessita di un maggior impegno a breve termine da parte degli operatori, pur garantendo a medio e lungo termine notevoli vantaggi sia per il paziente sia per la struttura sanitaria.

Per quanto riguarda il paziente, $\mathrm{i}$ vantaggi della EDD sono intuibili:

- Scomparsa del rischio di contrarre infezioni nosocomiali, che rappresentano tuttora una delle principali cause di morbidità e mortalità nella popolazione dialitica.

- Migliore riabilitazione socio-lavorativa, in quanto la seduta dialitica a domicilio può essere pianificata in accordo con le esigenze del lavoro e del tempo libero, può con- sentire il contemporaneo svolgimento di altre attività (ludiche e/o lavorative) e permette di evitare $i$ tempi morti per le attese e il trasporto al Centro dialisi.

- Miglior recupero psicologico, in particolare per quei pazienti che vivono in modo traumatico l'obbligo del legame con la struttura ospedaliera.

- Possibilità di incrementare il ritmo settimanale delle sedute dialitiche, ottenendo una migliore depurazione e correzione della ritenzione idrosodica (il che può consentire una maggiore libertà dietetica), quindi migliore benessere e sopravvivenza con ulteriori ricadute positive sullo stato psico-fisico del paziente.

Questi benefici possono controbilanciare il sovraccarico di impegno per la coppia.

Per quanto riguarda la struttura sanitaria, i vantaggi sono:

- Di ordine gestionale, perché la EDD consente di ovviare, almeno in parte, sia alla scarsità dei posti dialisi ospedalieri e ad assistenza limitata sia alla carenza di personale infermieristico, che rappresenta un problema emergente.

- Di ordine economico, in quanto consente di ridurre la spesa per il personale infermieristico.

Una delle ragioni che spiegano il ridotto avvio alla EDD è il cambiamento nella tipologia dei pazienti, dovuto sia alla prevalenza sempre maggiore di pazienti critici sia all'espansione dei programmi di trapianto per i pazienti in buone condizioni cliniche. Tuttavia, una volta accertata l'idoneità della coppia e l'esistenza delle idonee condizioni logistiche relative all'abitazione, bisogna espletare ogni tentativo per far emergere e per rafforzare le motivazioni che indirizzano al trattamento domiciliare.

Con questi criteri, meglio precisati in seguito, nella nostra unità operativa abbiamo mantenuto negli ultimi anni una percentuale pressoché costante (8-10\%) di pazienti in EDD che, se pur esigua in senso assoluto, è tra le più alte nella nostra Regione, e condizionata dal programma di trapianto che coinvolge in modo particolare questo gruppo di pazienti.

Per di più, tutti i pazienti in EDD nel nostro Centro praticano l'emodiafiltrazione ad alti flussi con UF controllata, metodica che abitualmente viene considerata di esclusiva applicazione nei Centri ospedalieri e nei CAL. 
Nella nostra esperienza, l'iter operativo da seguire per avviare un paziente alla EDD è il seguente:

- Offrire al potenziale paziente e al partner un'informazione corretta e completa sulla fattibilità della dialisi domiciliare e sugli indiscutibili vantaggi che ne derivano, anche attraverso contatti con pazienti già inseriti nel programma domiciliare. Contemporaneamente, svolgere un'azione di convincimento e di rassicurazione nei confronti del partner, che di solito è l'elemento che manifesta maggiori perplessità e timori. Il partner, che nella nostra opinione deve essere rappresentato da un congiunto (genitore, coniuge, e in casi particolari fratello o sorella), deve essere anche informato sulla possibilità di svolgere altre attività all'interno dell'abitazione durante la seduta dialitica. Ovviamente, è necessario informare sulle modalità del supporto che il Centro garantisce costantemente per qualsiasi problema logistico o clinico, come sarà dettagliato in seguito.

- Designare uno staff infermieristico che associ alla professionalità capacità didattiche e comunicative, e un medico referente che abbia una conoscenza complessiva delle problematiche inerenti al programma.

- Addestrare non più di una coppia alla volta, per evitare problemi di competitività che potrebbero inficiare l'efficacia dell'apprendimento. Naturalmente, questo comporta un sottoutilizzo dello staff infermieristico che però, a medio e lungo termine, si traduce in un vantaggio per il servizio.

- Iniziare l'addestramento dapprima solo rivolto al paziente in maniera da infondergli tranquillità e sicurezza riguardo al trattamento, e predisporre l'addestramento del partner (al momento in cui entra nel programma) congiuntamente da parte del paziente e dell'infermiere. Questa strategia ha lo scopo di favorire l'approccio del partner alla procedura anche attraverso la constatazione della sicurezza acquisita dal paziente.
- Non fissare limiti temporali rigidi per il training, per evitare l'ansia dell'apprendimento in tempi prefissati. Nella nostra esperienza l'autonomia della coppia è raggiunta in media in quattro mesi. È buona norma che la prima seduta dialitica a domicilio sia assistita anche dall'infermiera che ha condotto l'addestramento, per dare la sicurezza della continuità fra training e trattamento domiciliare.

- Garantire costantemente il massimo supporto gestionale e clinico, in particolare attraverso:

a) l'attivazione di una linea telefonica dedicata e, se possibile, un collegamento informatico;

b) la massima efficienza nel rifornimento del materiale e nello smaltimento dei rifiuti;

c) disponibilità da parte del Centro a trattare il paziente (con o senza partner) nell'arco della giornata e per tutto il tempo necessario, non solo per problemi clinici o tecnici, ma anche per garantire al partner, se richiesto, un periodo di riposo;

d) è opportuno un controllo clinico almeno mensile che includa esami ematochimici, visita generale e valutazione dell'aderenza alle prescrizioni dialitiche; a cadenza regolare, come per tutti i pazienti in dialisi cronica, controllo degli opportuni esami strumentali.

- Individuare tempestivamente, da parte del medico, eventuali segnali di stanchezza o di insofferenza nel partner e cercare di identificarne le cause per porvi rimedio nei limiti del possibile. Tale atteggiamento può contribuire alla prosecuzione del programma domiciliare il cui fallimento, nella maggior parte dei casi, è dovuto al manifestarsi o all'accentuarsi nella coppia di conflitti cui la dialisi domiciliare può contribuire.

In conclusione, non crediamo di avere fornito elementi di riflessione particolarmente nuovi riguardo al programma dialisi extracorporea domiciliare; abbiamo cercato, però, di ribadire i vantaggi di questo trattamento, di fornire le indicazioni pratiche scaturite dalla nostra espe- rienza e di sottolineare il ruolo centrale della figura del partner per la buona riuscita del programma.

dialisi@mbox.asl21.piemonte.it 